\title{
Myc Function in Drosophila
}

\author{
Peter Gallant \\ Julius-Maximilians-Universität Würzburg, Lehrstuhl für Biochemie und Molekularbiologie, Am Hubland, \\ 97074 Würzburg, Germany \\ Correspondence: peter.gallant@biozentrum.uni-wuerzburg.de
}

Drosophila contains a single MYC gene. Like its vertebrate homologs, it encodes a transcription factor that activates many targets, including prominently genes involved in ribosome biogenesis and translation. This activity makes Myc a central regulator of growth and/or proliferation of many cell types, such as imaginal disc cells, polyploid cells, stem cells, and blood cells. Importantly, not only does Myc act cell autonomously but it also affects the fate of adjacent cells and tissues. This potential of Myc is harnessed by many different signaling pathways, involving, among others, Wg, Dpp, Hpo, ecdysone, insulin, and mTOR.

D rosophila is the first species in which a $M y c$ gene has been described-albeit not under this name. In 1935, Calvin Bridges isolated a fly mutation that he called "diminutive" (Bridges 1935) that was later shown to correspond to a hypomorphic mutation of Drosophila Myc (Gallant et al. 1996; Schreiber-Agus et al. 1997). Whole-genome sequencing subsequently confirmed that the fly genome codes for a single MYC paralog, single MAX, and MXD family members (for review, see Gallant 2006) as well as MLX and Mondo/ChREBP orthologs. This lack of genetic redundancy of the dMyc/dMax/ dMnt (MXD) network, in combination with the large and ever-growing genetic toolkit and the vast literature on its biology, makes Drosophila an extremely valuable system for studying MYC function.

\section{THE Myc NETWORK IN DROSOPHILA}

The single Drosophila Myc protein (called "dMyc" below to distinguish it from the verte- brate Myc proteins; in the literature, both "Myc" and "dMyc" are used interchangeably) is equally similar to all vertebrate MYC paralogs, with a moderate overall sequence identity of $26 \%$ (for review, see Gallant 2006). However, the characterized domains are highly conserved. These include Myc Box 2, a centrally located acidic stretch that is variably known as Myc Box 3 or Myc Box 4, and the basic-helix-loop-helix leucine zipper domain. Only Myc Box 1 shows poor sequence conservation (Gallant et al. 1996; Schreiber-Agus et al. 1997). The functional properties of $\mathrm{dMyc}$ are also conserved. Thus, dMyc does not homodimerize, but specifically binds to (Drosophila and vertebrate) MAX. These dMyc:MAX heterodimers bind to the canonical MYC-binding site "CACGTG" (called "E box") and activate transcription from nearby genes (Gallant et al. 1996; Hulf et al. 2005). Moreover, dMyc can transform rodent fibroblasts after coexpression with vertebrate Ras ${ }^{\mathrm{V} 12}$ (Schreiber-Agus et al. 1997). dMyc can even substitute for MYC in murine embryo fibro-

Editors: Chi V. Dang and Robert N. Eisenman

Additional Perspectives on MYC and the Pathway to Cancer available at www.perspectivesinmedicine.org

Copyright (C) 2013 Cold Spring Harbor Laboratory Press; all rights reserved; doi: 10.1101/cshperspect.a014324 Cite this article as Cold Spring Harb Perspect Med 2013;3:a014324 
P. Gallant

blasts (Trumpp et al. 2001). Conversely, a human MYC variant rescues the lethality of a strong $d M y c$ mutant allele (Benassayag et al. 2005).

dMyc's binding partner MAX is also encoded by a single gene in the fly genome, and it is more highly conserved in its protein sequence (49\% overall identity to human MAX) (Gallant et al. 1996). To minimize confusion, here we use the name dMax for the fly protein and gene (in the literature, "Max" is also used). Although dMax is essential for Drosophila development and for dMyc's ability to transactivate E-boxcontaining target genes, some of dMyc's activities do not depend on the association with dMax (see section on Imaginal Discs) (Steiger et al. 2008). Besides binding to dMyc, dMax can homodimerize and interact with the single Drosophila member of the MAD/MXD/MNT protein family called dMnt (Peyrefitte et al. 2001; Loo et al. 2005). As in vertebrates, dMnt:dMax heterodimers repress the transcription of many dMyc:dMax-activated genes and thereby antagonize dMyc function (Loo et al. 2005; Pierce et al. 2008). In contrast to dMyc and dMax, $\mathrm{dMnt}$ is not essential for fly development (Loo et al. 2005).

Besides the MAX network, a parallel network centered on the MAX-like protein MLX has been identified in vertebrates (for review, see Billin and Ayer 2006). The components of this MLX network are also represented by single genes in Drosophila called bigmax (homologous to vertebrate MLX) and Mio (homologous to MondoA and MondoB in vertebrates). This network is discussed in O'Shea and Ayer (2013).

The following discussion focuses on $\mathrm{dMyc}$, which is by far the best-investigated member of the $\mathrm{dMyc} / \mathrm{dMax} / \mathrm{dMnt}$ network in Drosophila.

\section{dMyc Regulates Body Size}

dMyc's most obvious function is control of organismal size. When dMyc is ubiquitously (but moderately) overexpressed, flies grow to larger than normal adult sizes (de la Cova et al. 2004). Conversely, hypomorphic $d M y c$ mutations reduce the rate of larval growth, prolong the duration of development, and result in small adult flies (Gallant et al. 1996; Schreiber-Agus et al. 1997; Johnston et al. 1999). The complete elimination of dMyc activity (in a null mutation) blocks larval growth, such that the animals die in early development, usually barely larger than freshly hatched larvae (Pierce et al. 2004). This overall (organismic) size effect can be explained by dMyc's growth-promoting influence on various tissues in which dMyc is assumed to stimulate ribosome biogenesis and protein translation. Because the manifestation of this activity of dMyc differs among cell types, the different tissues are discussed separately below (see sections on Imaginal Discs, Polyploid Tissues, Stem Cells, and Blood Cells). A graphical overview of these activities is shown in Fig. 1. For simplicity's sake, the signaling pathways and upstream regulators that control $\mathrm{dMyc}$ activity are discussed for all tissues together.

\section{Imaginal Discs}

Imaginal discs consist of a monolayer of diploid columnar epithelial cells, whose proliferation is controlled by similar pathways as that of vertebrate cells. A second layer of squamous diploid cells (the peripodial membrane) that also contributes to imaginal discs is often neglected for experimental analysis. These discs are the precursors of most adult body wall structures (such as wings, thorax, legs, head and eyes, and antennae). During metamorphosis, they transform their morphology to adopt the adult organ shapes.

Overexpression of $\mathrm{dMyc}$ in imaginal cells strongly induces cellular growth; it increases cell size and accelerates their passage through $\mathrm{G}_{1}$ phase of the cell cycle (Johnston et al. 1999). However, the overall division rates of these cells are not increased. The cell cycle regulator $\mathrm{Cdc} 25$ / String becomes limiting for entry into $\mathrm{M}$ phase and, as a consequence, $G_{2}$ phase is extended. Only after coexpression of Cdc25/String is dMyc-induced excessive growth accompanied by increased proliferation (Johnston et al. 1999). Conversely, hypomorphic $d M y c$ mutant cells are characterized by a reduced growth rate and correspondingly smaller size. Proliferation rate is only affected when dMyc levels are strongly 


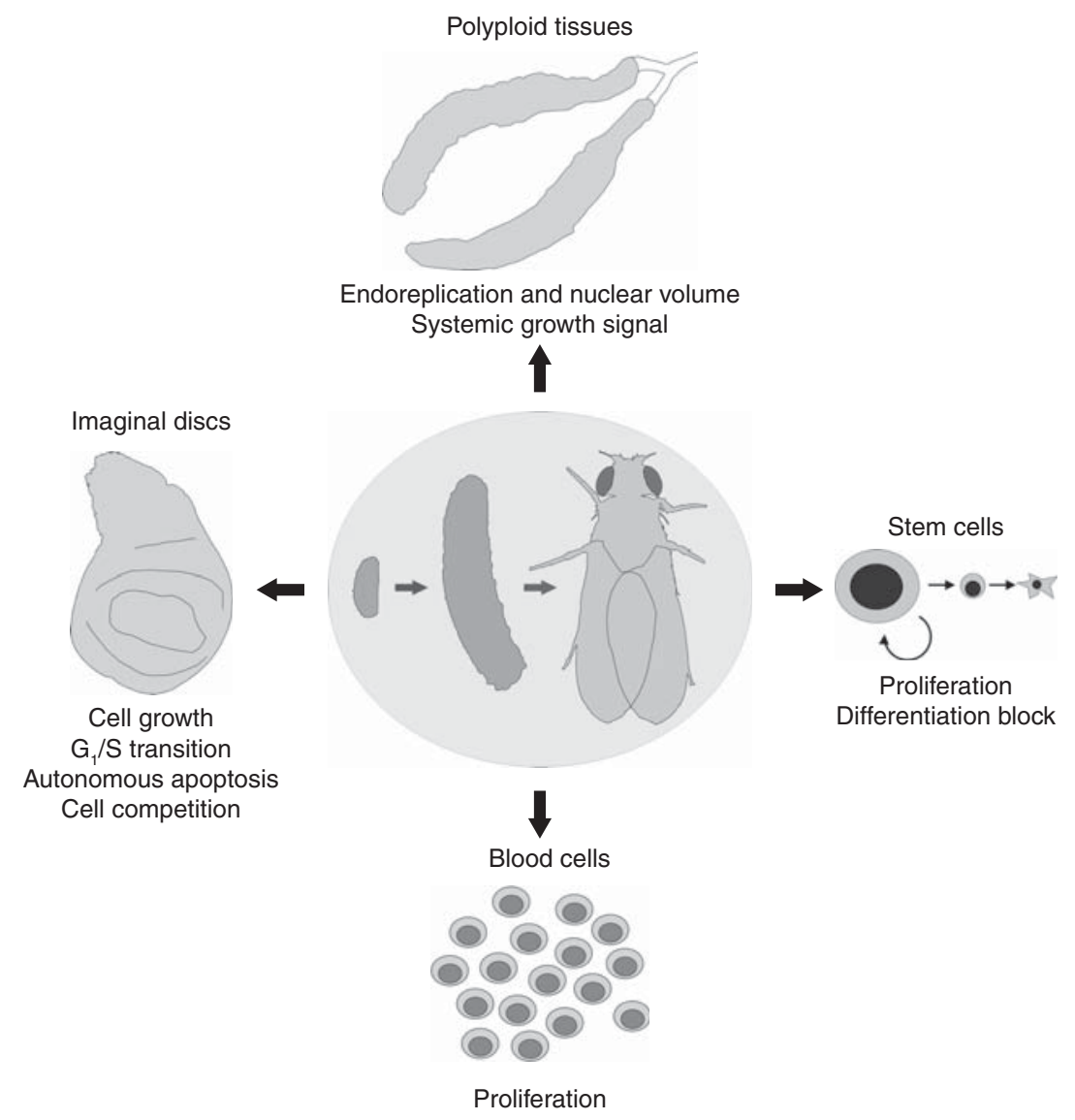

Figure 1. dMyc controls ribosome biogenesis and protein synthesis, and as a consequence, the accumulation of cellular mass and animal growth. Depending on tissue type, dMyc's activity primarily affects cell number, cell size, or cell fate. See text for details.

reduced. However, even $d M y c$ null mutant cells retain substantial growth capacity. This can be seen in flies that lack both $\mathrm{dMyc}$ and dMnt function; such animals grow slowly and never reach the adult stage, but a third of them develop to third instar larvae (and even pupae) that contain normally patterned imaginal discs (Pierce et al. 2008). Similarly, when dMyc (but not dMnt) activity is eliminated from wing or eye imaginal discs only, some adult animals develop that carry normally shaped, albeit small, organs (Schwinkendorf and Gallant 2009; Wu and Johnston 2010). These observations also indicate that normal organ patterning can take place in the absence of dMyc. The converse is also true: Moderate ubiquitous $\mathrm{dMyc}$ overexpression allows the development of normally patterned adult flies, even in a $d M y c$ null mutant background in which the transgene is the only source of $\mathrm{dMyc}$ (de la Cova et al. 2004; Schwinkendorf and Gallant 2009; Wu and Johnston 2010). In light of this lack of effect of dMyc levels on tissue patterning, it is interesting to note that normal dMyc expression is dynamically controlled by various patterning factors (Dpp, Wg, Notch; see sections on The Dpp Pathway and The Wg Pathway). It appears that this dynamic expression pattern is not crucial for dMyc's function as a growth promoter during normal development and that $\mathrm{dMyc}$ does not have an instructive role in patterned growth (Wu and Johnston 2010). In contrast, dMyc seems to be more important in 
modulating overall organ size in response to organ-extrinsic signals. Thus, the larva's nutritional status (i.e., amount and quality of available food) influences insulin/insulin-like growth factor signaling (IIS)/Tor-signaling strength, which in turn controls dMyc activity (see section on The dInr/mTOR Pathway). In addition, damage to imaginal discs (by mechanical or other means) triggers regenerative growth that is accompanied by, and critically dependent on, elevated dMyc levels (Smith-Bolton et al. 2009). Note that similar observations were also reported for certain stem cells (see section on Stem Cells).

Besides promoting the growth of imaginal cells, dMyc activity also correlates with the ability of these cells to activate an apoptosis program. High-level overexpression of dMyc induces apoptosis in the affected cells, via the induction of one or more of the proapoptotic genes hid, grim, reaper, and sickle (de la Cova et al. 2004; Montero et al. 2008; Schwinkendorf and Gallant 2009). Conversely, cells with reduced levels of dMyc are impaired in their ability to trigger apoptosis after exposure to low levels of DNA-damaging irradiation (Montero et al. 2008). In these settings, dMyc affects apoptosis cell autonomously.

In a different process, $\mathrm{dMyc}$ also influences apoptosis non-cell-autonomously. When $d M y c$ mutant cells are juxtaposed with wild-type cells in the same imaginal discs, the slower-growing $d M y c$ mutant cells are actively eliminated by their faster-growing wild-type neighbors (Johnston et al. 1999). This phenomenon is called cell competition. It was first described for cells carrying mutations in ribosomal protein genes (Morata and Ripoll 1975). Subsequently, it was also shown to be triggered by cells that mildly overexpress $\mathrm{dMyc}$ - in this case, the adjacent, slower-growing, wild-type cells are eliminated (de la Cova et al. 2004; Moreno and Basler 2004). Cell competition is thought to contribute to organ size control and optimize fitness by eliminating slowly growing, potentially defective cells. What is currently known about the molecular basis of this process, as well as its possible implications for human cancer, is discussed in Johnston (2013) and will therefore not be further addressed here.

\section{Polyploid Tissues}

Several larval tissues are made up of cells that stop dividing early during development and afterward endoreplicate their genomes until they reach ploidies of up to $1000 \mathrm{n}$, for example, salivary glands, fat body, muscles (whose ploidy is further increased by the fusion of several precursor cells). Similarly, the nurse cells that are derived from female germline stem cells and the somatic follicle cells that surround the developing egg chambers become polyploid through repeated endoreplication cycles. The increased DNA content endows these cells with a large biosynthetic capacity (Edgar and Orr-Weaver 2001). During metamorphosis, most of the larval polyploid tissues will be histolyzed and their content used for the developing adult tissues.

dMyc activity strongly influences the rate of endoreplication in all examined polyploid tissues. Whereas the cytoplasmic volume of these cells is only moderately affected by dMyc (Britton et al. 2002), dMyc overexpression dramatically increases their DNA content and nuclear volume. dMyc knockdown or depletion has the opposite outcome (Maines et al. 2004; Pierce et al. 2004; Quinn et al. 2004; Berry and Baehrecke 2007; Pierce et al. 2008; Demontis and Perrimon 2009).

This effect is presumably mediated by dMyc's "typical" transcriptional targets that affect ribosome biogenesis and translation rate (see section on Transcriptional Targets Mediating dMyc's Biological Functions). However, a transcription-independent direct action of $\mathrm{dMyc}$ on DNA replication, as has been suggested for vertebrate MYC (Dominguez-Sola et al. 2007), has not been ruled out.

In addition to the strictly cell-autonomous effects described in the sections Imaginal Discs and Polyploid Tissues above, manipulation of dMyc levels can also systemically impact the growth of the whole larva. Reduction of dMyc activity in larval muscles (by RNA interference or by overexpression of $\mathrm{dMnt}$ ) decreases the size of these muscles. As a consequence, the animal is physically impaired in its feeding ability, resulting in a diminished growth rate and overall size (Demontis and Perrimon 2009). This 
"dMyc effect" may appear to be trivial, but the situation is different for dMyc's activity in the fat body. Manipulations of dMyc levels in this polyploid tissue also induce cell-size changes that are paralleled by corresponding changes in overall larval size (Delanoue et al. 2010). However, changes in fat body cell size per se are not sufficient to affect larval growth, because Inr signaling in the fat body also autonomously controls cell size, but has no effect on overall larval size (Delanoue et al. 2010). Hence, it has been proposed that $\mathrm{dMyc}$ has a role in the production of a fat-body-derived signal. This signal promotes systemic growth, at least partly, by triggering the secretion of insulin-like peptides from dedicated neurosecretory cells (for review, see Rajan and Perrimon 2011). Besides $\mathrm{dMyc}$, a limited number of other factors have been shown to contribute to the synthesis of this fat-body-derived signal, most notably Tor (Colombani et al. 2003); the transcription factor DREF, which may activate several genes involved in ribosome biogenesis and function (Killip and Grewal 2012); and RNA polymerase III (Marshall et al. 2012; Rideout et al. 2012). All of these have been linked to dMyc activity. Thus, the availability of nutrients ( particularly amino acids) influences Tor activity, which controls $\mathrm{dMyc}$ protein levels in the fat body. dMyc is an essential downstream effector of Tor (see section on The dInr/mTOR Pathway). dMyc, in turn, activates the expression of DREF (Thao et al. 2008; Killip and Grewal 2012) and additionally may cooperate with DREF in the activation of common target genes (Orian et al. 2003). dMyc also activates RNA polymerase III, in part by increasing the expression of its cofactor Brfl (Marshall et al. 2012), and in part by physically interacting with Brf1 (Steiger et al. 2008; Marshall et al. 2012). The fat-body-derived signal itself has recently been shown to be the cytokine Upd2/Leptin (Rajan and Perrimon 2012)_or rather, one of the fat-body-derived signals, because there may be more than one. It is currently unclear how Upd 2 production or release from fat body cells might be affected by dMyc.

Taken together, these findings outline a physiological pathway connecting nutrition with systemic animal growth, in which $\mathrm{dMyc}$ has a central role in the production of a nonautonomous signal.

\section{Stem Cells}

dMyc has also been investigated in tissues that derive from precursor cells with stem-cell-like properties: the ovary (Jin et al. 2008; Neumuller et al. 2008; Rhiner et al. 2009) and adult gut (Amcheslavsky et al. 2011; Cordero et al. 2012a, 2012b). In addition, some similar observations were made for the developing nervous system (Betschinger et al. 2006; Song and Lu 2011). In all of these tissues, dMyc protein levels are high in the stem cells but diminish in the differentiating daughter cells that result from asymmetric cell division. This drop in $\mathrm{dMyc} \mathrm{lev-}$ els is functionally important because forced overexpression of dMyc prevents the normal differentiation of the daughter cells in the ovary (cystoblasts) (Rhiner et al. 2009). A similar observation was made in the adult gut. This tissue consists of intestinal stem cells (ISCs) that divide to produce enteroblasts (EBs) that, in turn, give rise to differentiated enterocytes. dMyc overexpression in ISCs enhances the consequences of Tsc2 knockdown and ensuing mTOR activation, that is, a block of differentiation of the EBs. Curiously, dMyc also prevents stem cell proliferation in this latter setting (Amcheslavsky et al. 2011). Conversely, after dMyc is depleted from ISCs for several days, their proliferation arrests and gut homeostasis is impaired (Amcheslavsky et al. 2011). The requirement for $\mathrm{dMyc}$ is more pronounced during gut regeneration. After damage (such as what is caused by ingestion of the DNA-damaging drug bleomycin), dMyc levels are up-regulated in the ISCs and their proliferation is stimulated. If $\mathrm{dMyc}$ induction is dampened by knockdown, or even by heterozygosity for a $d M y c$ null allele, this ISC proliferation burst is prevented and the damage cannot be repaired (Cordero et al. 2012b). This involvement of dMyc is reminiscent of dMyc's important role in imaginal disc regeneration (see section on Imaginal Discs). Interestingly, MYC has also been proposed to have a role in tissue regeneration in vertebrates, in which MYC levels strongly increase after partial hepatectomy in 
P. Gallant

rats (Goyette et al. 1984). However, the functional relevance of this MYC induction is unclear (Baena et al. 2005; Li et al. 2006).

An analogous pronounced requirement for $\mathrm{dMyc}$ was recently also reported for the growth of larval neuroblasts under pathological conditions (Song and Lu 2011). Depletion of dMyc from normally developing neuroblasts had no noticeable effect on their maintenance. However, ectopic neuroblasts, which could be induced by various tumorigenic mutations, were efficiently suppressed by dMyc knockdown. In this situation, dMyc was shown to promote the expression of the translation factor eIF$4 \mathrm{E}$, which acted in a feedback loop to stimulate the transcriptional activity of $\mathrm{dMyc}$ (Song and Lu 2011). Thus, as for gut ISCs and imaginal disc regeneration, dMyc appears to be particularly important under conditions of excessive growth.

In ovarian stem cells, the down-regulation of dMyc leads to a disadvantage in the competition for niche occupancy, according to one recent publication (Rhiner et al. 2009). A stem cell niche contains two to three germline stem cells (GSCs), and GSCs with comparatively lower $\mathrm{dMyc}$ levels are lost from the niche and replaced by their sisters. Hence, attaining sufficiently high dMyc levels is also a prerequisite for maintaining the stem cell population (Rhiner et al. 2009). However, a different group observed no such dMyc-dependent competition between GSCs (Jin et al. 2008).

These observations suggest a correlation between elevated dMyc levels and the "stemness" of progenitor cells in at least some tissues, and they reinforce the notion that $\mathrm{dMyc}$ has an important role in allowing cells to adapt to ectopic signals. (See also Chappell and Dalton [2013] for an extensive discussion of MYC's function in vertebrate stem cells.)

\section{Blood Cells}

The most frequently used Drosophila cell line, Schneider S2 cells, is derived from embryonic macrophages. Depletion of $\mathrm{dMyc}$ from these cells reduces their size (Hulf et al. 2005) and proliferation rate (Boutros et al. 2004; Bjorklund et al. 2006), consistent with dMyc's effect on imaginal disc cells in vivo (Johnston et al. 1999).

A recent study has shown that $\mathrm{dMyc}$ also controls the proliferation of blood cell precursors in third instar larval lymph glands in vivo (Pennetier et al. 2012). In control animals, dMyc is expressed at high levels in the proliferative compartment of the lymph gland. Pennetier and coworkers raised these levels even further, either by dMyc overexpression or down-regulation of Dpp signaling (which normally inhibits $\mathrm{dMyc}$ expression and proliferation in this particular tissue). Both treatments resulted in overproliferation in this compartment and prevented the differentiation of precursor cells (located in the lymph gland) to mature blood cells. Conversely, dMyc knockdown abrogated the overproliferation phenotype that was caused by inhibition of Dpp signaling. Possible effects of dMyc on cell size were not addressed in this study (Pennetier et al. 2012).

Taken together, these observations indicate that dMyc controls cellular growth, proliferation, death, and differentiation in many (possibly all) tissues. Depending on the cell type, changes in dMyc activity primarily affect cell number or cell size. It is conceivable that these different outcomes reflect different biological activities of $\mathrm{dMyc}$, but it is equally possible that $\mathrm{dMyc}$ primarily promotes growth and that the observed outcomes depend on the "wiring" of a particular cell type that links growth to division.

\section{Transcriptional Targets Mediating dMyc's Biological Functions}

Like its vertebrate homologs (Eilers and Eisenman 2008), dMyc acts as a transcription factor (Gallant et al. 1996). Vertebrate MYC has been reported to also possess nontranscriptional activities, that is, transcription-independent control of DNA replication (Dominguez-Sola et al. 2007) and acetylation of microtubules (Conacci-Sorrell et al. 2010). However, these functions have not been addressed in flies, and attempts to understand dMyc's biological activity have focused on its transcriptional targets in various cell types: $\mathrm{S} 2$ cells, imaginal discs, fat bodies 
and muscles, and whole larvae (Orian et al. 2003; Grewal et al. 2005; Hulf et al. 2005; Li et al. 2010). Although these studies used different technologies (DamID, spotted microarrays, Affymetrix microarrays) and are therefore not directly comparable, similar conclusions emerged from all of them. Thus, dMyc controls many genes in flies, possibly hundreds, but there is no indication (yet) that it acts as a general transcriptional amplifier, as has been proposed for vertebrate MYC (Lin et al. 2012; Nie et al. 2012).

The most prominent group of dMyc targets is involved in ribosome biogenesis (Teleman et al. 2008; Li et al. 2010). Many of the genes in this class are homologs of bona fide vertebrate MYC targets, and several of them have been individually confirmed as essential growth mediators downstream from $\mathrm{dMyc}$, for example, pitchoune (DDX18 in vertebrates) (Zaffran et al. 1998), modulo (nucleolin in vertebrates) (Perrin et al. 2003), and viriato (Nol12 in vertebrates) (Marinho et al. 2011). This group of genes is characterized by the presence of canonical dMyc-binding sites, typically located within the first 50 nucleotides downstream from the transcription start site. Presence and position of these E boxes strongly argue that these genes constitute direct $\mathrm{dMyc}$ targets and that $\mathrm{dMyc}$ might control their expression at a postinitiation stage (Hulf et al. 2005; Teleman et al. 2008; Li et al. 2010). In addition to these protein-coding genes, dMyc also stimulates the RNA polymerase I- and RNA polymerase III-dependent transcription of ribosomal RNA and small noncoding RNAs (e.g., tRNAs and 5S rRNA), respectively. The former effect is indirect, via dMyc's induction of several RNA polymerase I cofactors, such as TIF-1A (Grewal et al. 2005). In the case of RNA polymerase III (Pol III), dMyc both activates the expression of the cofactor Brf1 (Marshall et al. 2012) and physically interacts with Brf1 to directly stimulate Pol III activity (Steiger et al. 2008; Marshall et al. 2012). Interestingly, dMyc's interaction with Brf1 and activation of RNA Pol III do not require association with dMax, and therefore dMax null mutant larvae retain $\mathrm{dMyc}$-dependent control of Pol III activity. This is one reason (although maybe not the only one) why dMax mutant animals grow substantially better than $d M y c$ null mutants (Steiger et al. 2008). In addition to the targets mentioned above, dMyc also activates genes coding for ribosomal proteins and translation factors (Orian et al. 2003; Hulf et al. 2005). These genes often do not contain canonical E boxes ( Li et al. 2010). In some cases, their induction by dMyc may be indirectly mediated by the transcription factor DREF, which itself is a dMyc target (Thao et al. 2008; Killip and Grewal 2012) or by dMyc in conjunction with DREF (Orian et al. 2003). These targets act in concert to control protein synthesis and cellular growth, and hence, they go a long way toward explaining dMyc's growth-promoting activity. Many of them function in the nucleolus to control processing and assembly of ribosomal components. Accordingly, increased dMyc activity is reflected in enlarged nucleolar volumes, which can be seen particularly well in polyploid cells (Grewal et al. 2005; Marinho et al. 2011). Note that such enlarged nucleoli have also long been observed in mammalian tumors (Ruggero 2012). The concerted regulation of this entire set of "growth genes" by dMyc is exploited by the animal when it needs to adapt to varying environmental conditions. Thus, starvation of Drosophila larvae triggers the inhibition of the central growth regulator mTOR, which in turn leads to the destabilization of dMyc protein (see section on The dInr/mTOR Pathway). The ensuing collective repression of these target genes, and a concomitant reduction in growth rate (Teleman et al. 2008; Li et al. 2010). Conversely, tissue regrowth after damage reactivates protein synthesis and this is synchronized by up-regulation of $\mathrm{dMyc}$ (see sections on Imaginal Discs and Stem Cells above) (Smith-Bolton et al. 2009; Cordero et al. 2012b). This intimate connection between $\mathrm{dMyc}$ and the protein synthesis machinery is also illustrated by the observation that, after experimental depletion of different translation factors, the cell apparently compensates by upregulating dMyc expression (Bonke et al. 2013).

Besides these "growth genes," dMyc also controls the expression of several cell cycle regulators. Thus, dE2F1, RBF, Stg/Cdc25, several cyclins, and Cdks have been proposed to be transcriptionally controlled by dMyc (Orian et al. 
P. Gallant

2003; Duman-Scheel et al. 2004; Hulf et al. 2005) and cyclin $\mathrm{E}$ at the posttranscriptional level (via an unknown mechanism) (Prober and Edgar 2000). Moreover, dMyc may contribute to the expression of histone genes, as suggested by the localization of $\mathrm{dMyc}$ protein to the sites of histone gene transcription in embryos, to so-called "histone locus bodies" (Daneshvar et al. 2011). Taken together, these observations suggest that some of dMyc's impact on cellular proliferation could be direct and not an indirect consequence of the dMyc stimulated growth.

The list of dMyc targets contains many more than those genes mentioned above. However, beyond these aforementioned genes few have been linked to specific biological outcomes. Thus, in young female embryos, dMyc has an important role in activating the switch gene $S x l$ and thereby fixing the sex of such zygotes (Kappes et al. 2011). In addition, in embryos, dMyc interacts with the corepressor Groucho to repress a set of proneural Notch target genes, antagonizing neuronal differentiation (Orian et al. 2007). Finally, in conjunction with dMax, dMyc autorepresses its own expression, analogous to what has been reported for vertebrate MYC (Goodliffe et al. 2005; Steiger et al. 2008).

Because of space restrictions, the mechanisms by which dMyc regulates target gene expression cannot be addressed here. Suffice it to say that several proteins have been shown to assist Myc in gene repression (Pontin and the Polycomb-group proteins Pc, pho, and Psc) (Bellosta et al. 2005; Goodliffe et al. 2005, 2007) or activation (dHCF and the trithorax-group proteins ash1 and Lid) (Goodliffe et al. 2005, 2007; Secombe et al. 2007; Furrer et al. 2010). Most of these regulatory interactions appear to be evolutionarily conserved in vertebrates. A prominent exception is the Myc-interacting transcription factor Miz1, which has not been found in flies, although it has a key role in Myc-dependent gene repression in vertebrates (see Wiese et al. 2013).

\section{CONTROL OF dMyc LEVELS}

Given dMyc's broad reach on growth and proliferation, it is to be expected that many signal- ing pathways harness dMyc's potential. Indeed, the last years have seen the identification of numerous factors influencing dMyc mRNA and protein levels. Selected examples of such signaling pathways are described below: the $\mathrm{Wg}$ and the Dpp pathways, which were originally characterized for their roles in pattern formation; the $\mathrm{Inr} / \mathrm{mTOR}$ and Hpo pathways, which are prominent regulators of growth; and the EcR pathway, which has a key role in timing developmental transitions.

\section{The Wg Pathway}

The secreted morphogen Wingless/Wg influences dMyc levels in several tissues. The underlying mechanisms depend on the context, and they are only partially understood. Thus, Wg represses $\mathrm{dMyc}$ expression in a group of wing imaginal disc cells that is located in the presumptive wing margin, the so-called zone of nonproliferating cells (ZNC). When Wg signaling is inhibited by expression of a dominantnegative version of TCF, an essential downstream component of the Wg signaling pathway, dMyc expression is reestablished (Johnston et al. 1999; Duman-Scheel et al. 2004). Two scenarios have been proposed to explain this negative effect of Wg on dMyc. According to the first, Wg induces the protein Hfp (homolog of vertebrate FIR, which was shown to repress MYC expression) (Quinn et al. 2004). Hfp then binds to the $\mathrm{dMyc}$ gene and inhibits the DNA helicase Hay/ $\mathrm{XBP}$, which is required for normal dMyc expression (Mitchell et al. 2010). In the second scenario, $\mathrm{Wg}$ is produced in the $\mathrm{ZNC}$ and diffuses to neighboring cells to activate the Notch ligands Delta and Serrate. This leads to the activation of the Notch pathway within the $\mathrm{ZNC}$ that then inhibits dMyc expression (Herranz et al. 2008). The extent to which these two pathways contribute to $\mathrm{dMyc}$ regulation in the $\mathrm{ZNC}$ is currently unclear.

In other settings, Wg has been reported to induce dMyc expression. Examples include other regions of normally developing wing discs (Herranz et al. 2008), wing discs that are regenerating after damage (Smith-Bolton et al. 2009), and possibly lymph glands (Pennetier et al. 
2012). An intriguing connection between $\mathrm{Wg}$ and dMyc was recently also observed in the adult gut. Experimental damage to the gut epithelium triggers the synthesis and release of Wg in EBs. Activation of the canonical Wg signaling pathway in the neighboring ISCs promotes the expression of $\mathrm{dMyc}$, which is essential for the proliferative burst of these cells and the ensuing regeneration of the damaged gut (Cordero et al. 2012a,b). Interestingly, a similar epistatic interaction between $\mathrm{dMyc}$ and $\mathrm{Wg}$ has previously also been shown for vertebrates, in which colon cancers induced by deregulated Wg signaling were shown to require induction of $\mathrm{dMyc}$ expression (Sansom et al. 2007).

\section{The Dpp Pathway}

Another secreted factor that regulates both patterning and growth in imaginal discs is Decapentaplegic/Dpp, the best-known Drosophila representative of the TGF- $\beta /$ BMP family (Affolter and Basler 2007). Dpp's growth effect can be explained by its ability to down-regulate the expression of the transcriptional repressor and growth inhibitor Brinker/Brk. Among many other targets, Brk binds to several sites in the $\mathrm{dMyc}$ gene. The resulting $d M y c$ repression and subsequent derepression by Dpp signaling substantially contribute to the Dpp-dependent growth stimulation (Doumpas et al. 2013).

In light of these findings, it is tempting to speculate that the high levels of Dpp signaling experienced by certain stem cells (e.g., female GSCs) might contribute to the high dMyc levels observed in these cells. Conversely, it has been proposed that dMyc activity in GSCs enhances the transduction of the Dpp signal, potentially contributing to a bistable loop-with GSCs displaying high intracellular Dpp signaling and dMyc levels and the differentiating cysts having neither (Harris et al. 2011).

\section{The Hpo Pathway}

Compared to the Wg and Dpp pathways, Hpo signaling has only recently been discovered, and it is mostly being analyzed for its effects on growth (as well as for the consequences of its deregulation on human cancer) (Pan 2010). Activation of this pathway prevents the nuclear translocation of the transcriptional cofactor Yorkie/Yki and restrains growth. Hpo signaling can be inhibited in wing imaginal disc cells by inactivation of the tumor suppressors that make up its core module, Hpo, Wts, Sav, and Mats. As a consequence, a complex of the sequence-specific transcription factor Sd (homologous to the vertebrate TEAD1-4) with Yki binds to several sites in dMyc's promoter and second intron and induces dMyc expression (Neto-Silva et al. 2010). dMyc is essential for Yki's ability to promote overgrowth, but it clearly is not the only important downstream effector. Other activated targets include the growth-promoting miRNA bantam and the caspase inhibitor Diap1.

Interestingly, in addition to acting downstream from Yki, dMyc requires Yki for its biological activity. In Yki mutant cells, dMyc cannot induce overgrowth, but coexpression with either of the Yki targets Diap1 or bantam partially overcomes this deficiency. Because dMyc overexpression does not increase Yki levels, but instead moderately down-regulates them, it is presumably the basal expression of Yki targets that is required for this $\mathrm{dMyc}$-dependent overgrowth (Neto-Silva et al. 2010).

Hpo signaling also controls dMyc levels in glial cells, but in this tissue the effect of Yki on $\mathrm{dMyc}$ is not direct. Instead, a complex of Yki with the transcription factor Mad activates the microRNA bantam, which then induces dMyc (Reddy and Irvine 2011). How bantam influences dMyc in the glia is not clear. However, a possible molecular path from bantam to dMyc is suggested by separate observations in other tissues. Thus, in wing discs, bantam was shown to down-regulate the ubiquitin ligase Mei-P26 (Herranz et al. 2010). On the other hand, MeiP26 and the related protein Brain tumor/Brat have both been shown to reduce $\mathrm{dMyc}$ protein levels in a number of tissues ( $\mathrm{S} 2$ cells, wing discs, female germline cells, and neurons) (Betschinger et al. 2006; Neumuller et al. 2008; Rhiner et al. 2009; Herranz et al. 2010; Harris et al. 2011). These ubiquitin ligases presumably act by blocking $\mathrm{dMyc}$ translation and/or by degrading $\mathrm{dMyc}$ protein, analogous to the reported ubiquitina- 
P. Gallant

tion and degradation of murine MYC by the Mei-P26/Brat homolog TRIM32 (Schwamborn et al. 2009). Mutational inactivation of Brat and Mei-P26 in neuroblasts and female GSCs, respectively, deregulates dMyc expression and results in tumorous overgrowth. Note that these observations are also consistent with the notion (described in the section Stem Cells) that downregulation of $\mathrm{dMyc}$ is required for the differentiation of stem cells.

\section{The dInr/mTOR Pathway}

A central part in cellular and organismal size control is played by the IIS pathway and the kinase mTOR. Inhibition of mTOR by the inhibitor rapamycin, genetic means, or starvation leads to posttranscriptional down-regulation of $\mathrm{dMyc}$ and the concomitant repression of $\mathrm{dMyc}$ target genes (Teleman et al. 2008; Li et al. 2010; Parisi et al. 2011). This effect has been shown for S2 cells and the fat body, but it also presumably occurs in other cell types (e.g., intestinal stem cells) (Amcheslavsky et al. 2011). This dMyc down-regulation is mediated in part by the kinases S6K and Sgg/GSK-3 $\beta$. Under optimal growth conditions, $\mathrm{S} 6 \mathrm{~K}$ is activated by $\mathrm{mTOR}$ and contributes to increased dMyc levels (Parisi et al. 2011). On the other hand, Sgg/GSK-3 $\beta$ is active under starvation conditions and then contributes to dMyc degradation in a process analogous to that described in vertebrates (Schulein and Eilers 2009): by phosphorylating dMyc (Galletti et al. 2009), GSK-3 $\beta$ creates a recognition site for the F-box protein ago/Fbw7, allowing it to ubiquitinate and degrade $\mathrm{dMyc}$ (Moberg et al. 2004). In the case of MYC, this degradation is promoted by an axin-containing complex (Arnold et al. 2009). Similarly, in Drosophila, the axin-associated kinases $\mathrm{CK} 1 \alpha / \varepsilon$ contribute to dMyc degradation (Galletti et al. 2009; Legent et al. 2012). Interestingly, one of these kinases, $\mathrm{CK} 1 \varepsilon$, was recently also found to genetically interact with MYC in vertebrate tumor cells, although the underlying mechanism has not been addressed (Toyoshima et al. 2012). It is tempting to speculate that CK1 family kinases might also mediate some of the effects of $\mathrm{Wg}$ on dMyc levels, given their established in- volvement in the Wg signaling pathway (Cheong and Virshup 2011).

The IIS pathway also stabilizes $\mathrm{dMyc}$ by inhibiting Sgg/GSK-3 $\beta$ (Parisi et al. 2011). In addition, IIS controls dMyc expression through the transcription factor dFoxo, which can directly bind to the $d M y c$ promoter (Teleman et al. 2008; Demontis and Perrimon 2009). However, the effects of dFoxo on dMyc expression are complex and depend on tissue type and nutritional status. In the fat body, dFoxo represses dMyc under optimal nutritional conditions (by an unclear mechanism) but activates dMyc on starvation. This helps to maintain a constant metabolic activity in the fat body, which thereby can serve in its role as a central support organ for the organism (Teleman et al. 2008). In contrast, in muscles dFoxo only represses dMyc on starvation ( possibly indirectly), thereby helping to avoid unnecessary energy expenditure (Teleman et al. 2008). Finally, dFoxo was also shown to inhibit dMyc's transcriptional activity when coexpressed in S2 cells (Demontis and Perrimon 2009).

\section{The EcR Pathway}

In contrast to the signaling pathways mentioned in the sections The Wg Pathway, The Dpp Pathway, The Hpo Pathway, and The dInr/mTOR Pathway, the molting hormone 20-hydroxy-ecdysone/20E is best known for its effects on the timing of developmental transitions. $20 \mathrm{E}$ binds to the nuclear hormone receptor EcR and thereby allows the EcR:Usp complex to control the transcription of its target genes. One of these targets is dMyc. Genome-wide-binding studies conducted in tissue culture cells found the EcR:Usp complex to bind to the $d M y c$ locus, and bioinformatic analysis revealed several potential EcR:Usp-binding motifs in $d M y c$ (Gauhar et al. 2009). In the fat body, the EcR:Usp complex was shown to repress $\mathrm{dMyc}$ expression (Delanoue et al. 2010; Kamoshida et al. 2012). This repression affects metabolism of the fat body and reduces lipid accumulation (Kamoshida et al. 2012). At the same time, EcR:Usp also reduces the amount of the dMyc-dependent fat-body-derived systemic growth signal 
and thereby diminishes overall larval growth (Delanoue et al. 2010). Thus, the hormone 20 E not only triggers developmental transitions (through its concentration peaks), but basal levels of $20 \mathrm{E}$ also affect animal growth rate-and the regulation of $\mathrm{dMyc}$ expression in the fat body has a central role in this process (Tennessen and Thummel 2011).

\section{CONCLUDING REMARKS}

Research on Drosophila Myc has spread over the last years to cover a large variety of molecular, cell-biological, and developmental aspects. Among the most important findings that resulted from this work, I would count to be the realization that $\mathrm{dMyc}$ controls cellular growth both autonomously as well as in a nonautonomous manner-notably by the process of cell competition. In addition, we have made significant progress in understanding the interaction of dMyc with different signaling pathways and its relevance for various biological processes, thanks to the availability of various reporters, the easy access to all tissues, and (last but not least) the Drosophila genetic toolkit. Importantly, based on past experience, I am optimistic that most of these findings will be relevant for human biology.

\section{ACKNOWLEDGMENTS}

Many thanks to Laura Johnston, Hugo Stocker, Bob Eisenman, and Chi Dang for their comments on the manuscript. Research in the author's laboratory is supported by grants from the Deutsche Forschungsgemeinschaft (DFG).

\section{REFERENCES}

${ }^{*}$ Reference is also in this collection.

Affolter M, Basler K. 2007. The Decapentaplegic morphogen gradient: From pattern formation to growth regulation. Nat Rev Genet 8: 663-674.

Amcheslavsky A, Ito N, Jiang J, Ip YT. 2011. Tuberous sclerosis complex and Myc coordinate the growth and division of Drosophila intestinal stem cells. J Cell Biol 193: 695-710.

Arnold HK, Zhang X, Daniel CJ, Tibbitts D, Escamilla-Powers J, Farrell A, Tokarz S, Morgan C, Sears RC. 2009. The
Axin 1 scaffold protein promotes formation of a degradation complex for c-Myc. EMBO J 28: 500-512.

Baena E, Gandarillas A, Vallespinos M, Zanet J, Bachs O, Redondo C, Fabregat I, Martinez AC, de Alboran IM. 2005. c-Myc regulates cell size and ploidy but is not essential for postnatal proliferation in liver. Proc Natl Acad Sci 102: 7286-7291.

Bellosta P, Hulf T, Balla Diop S, Usseglio F, Pradel J, Aragnol D, Gallant P. 2005. Myc interacts genetically with Tip48/ Reptin and Tip49/Pontin to control growth and proliferation during Drosophila development. Proc Natl Acad Sci 102: 11799-11804.

Benassayag C, Montero L, Colombie N, Gallant P, Cribbs D, Morello D. 2005. Human c-Myc isoforms differentially regulate cell growth and apoptosis in Drosophila melanogaster. Mol Cell Biol 25: 9897-9909.

Berry DL, Baehrecke EH. 2007. Growth arrest and autophagy are required for salivary gland cell degradation in Drosophila. Cell 131: 1137-1148.

Betschinger J, Mechtler K, Knoblich JA. 2006. Asymmetric segregation of the tumor suppressor brat regulates selfrenewal in Drosophila neural stem cells. Cell 124: 12411253

Billin AN, Ayer DE. 2006. The Mlx network: Evidence for a parallel Max-like transcriptional network that regulates energy metabolism. Curr Top Microbiol Immunol 302: 255-278.

Bjorklund M, Taipale M, Varjosalo M, Saharinen J, Lahdenpera J, Taipale J. 2006. Identification of pathways regulating cell size and cell-cycle progression by RNAi. Nature 439: 1009-1013.

Bonke M, Turunen M, Sokolova M, Vaharautio A, Kivioja T, Taipale M, Bjorklund M, Taipale J. 2013. Transcriptional networks controlling the cell cycle. G3 3: 75-90.

Boutros M, Kiger AA, Armknecht S, Kerr K, Hild M, Koch B, Haas SA, Consortium HFA, Paro R, Perrimon N. 2004. Genome-wide RNAi analysis of growth and viability in Drosophila cells. Science 303: 832-835.

Bridges CB. 1935. Drosophila melanogaster: Legend for symbols, mutants, valuations. Drosophila Information Service 3: $5-19$.

Britton JS, Lockwood WK, Li L, Cohen SM, Edgar BA. 2002. Drosophila's insulin/PI3-kinase pathway coordinates cellular metabolism with nutritional conditions. Dev Cell 2: 239-249.

* Chappell J, Dalton S. 2013. Roles for MYC in the establishment and maintenance of pluripotency. Cold Spring Harb Perspect Med doi: 10.1101/cshperspect.a014381.

Cheong JK, Virshup DM. 2011. Casein kinase 1: Complexity in the family. The Int J Biochem Cell Biol 43: 465-469.

Colombani J, Raisin S, Pantalacci S, Radimerski T, Montagne J, Leopold P. 2003. A nutrient sensor mechanism controls Drosophila growth. Cell 114: 739-749.

Conacci-Sorrell M, Ngouenet C, Eisenman RN. 2010. Mycnick: A cytoplasmic cleavage product of Myc that promotes $\alpha$-tubulin acetylation and cell differentiation. Cell 142: $480-493$.

Cordero JB, Stefanatos RK, Myant K, Vidal M, Sansom OJ. 2012a. Non-autonomous crosstalk between the Jak/Stat and Egfr pathways mediates Apcl-driven intestinal stem 
P. Gallant

cell hyperplasia in the Drosophila adult midgut. Development 139: 4524-4535.

Cordero JB, Stefanatos RK, Scopelliti A, Vidal M, Sansom OJ. 2012b. Inducible progenitor-derived Wingless regulates adult midgut regeneration in Drosophila. EMBO J 31: 3901-3917.

Daneshvar K, Khan A, Goodliffe JM. 2011. Myc localizes to histone locus bodies during replication in Drosophila. PLOS ONE 6: e23928.

de la Cova C, Abril M, Bellosta P, Gallant P, Johnston LA 2004. Drosophila myc regulates organ size by inducing cell competition. Cell 117: 107-116.

Delanoue R, Slaidina M, Léopold P. 2010. The steroid hormone ecdysone controls systemic growth by repressing dMyc function in Drosophila fat cells. Dev Cell 18: 1012-1021.

Demontis F, Perrimon N. 2009. Integration of insulin receptor/Foxo signaling and $\mathrm{dMyc}$ activity during muscle growth regulates body size in Drosophila. Development 136: $983-993$.

Dominguez-Sola D, Ying CY, Grandori C, Ruggiero L, Chen B, Li M, Galloway DA, Gu W, Gautier J, Dalla-Favera R. 2007. Non-transcriptional control of DNA replication by c-Myc. Nature 448: 445-451.

Doumpas N, Ruiz-Romero M, Blanco E, Edgar B, Corominas M, Teleman AA. 2013. Brk regulates wing disc growth in part via repression of Myc expression. EMBO Rep 14: 261-268.

Duman-Scheel M, Johnston LA, Du W. 2004. Repression of dMyc expression by Wingless promotes Rbf-induced G1 arrest in the presumptive Drosophila wing margin. Proc Natl Acad Sci 101: 3857-3862.

Edgar BA, Orr-Weaver TL. 2001. Endoreplication cell cycles: More for less. Cell 105: 297-306.

Eilers M, Eisenman RN. 2008. Myc's broad reach. Genes Dev 22: 2755-2766.

Furrer M, Balbi M, Albarca-Aguilera M, Gallant M, Herr W, Gallant P. 2010. Drosophila Myc interacts with host cell ractor (dHCF) to activate transcription and control growth. J Biol Chem 285: 39623-39636.

Gallant P. 2006. Myc/Max/Mad in invertebrates: The evolution of the Max network. Curr Top Microbiol Immunol 302: $235-253$.

Gallant P, Shiio Y, Cheng PF, Parkhurst SM, Eisenman RN. 1996. Myc and Max homologs in Drosophila. Science 274: $1523-1527$.

Galletti M, Riccardo S, Parisi F, Lora C, Saqcena MK, Rivas L, Wong B, Serra A, Serras F, Grifoni D, et al. 2009. Identification of domains responsible for ubiquitin-dependent degradation of dMyc by glycogen synthase kinase $3 \beta$ and casein kinase 1 kinases. Mol Cell Biol 29: 34243434 .

Gauhar Z, Sun LV, Hua S, Mason CE, Fuchs F, Li TR, Boutros M, White KP. 2009. Genomic mapping of binding regions for the Ecdysone receptor protein complex. Genome Res 19: 1006-1013.

Goodliffe JM, Wieschaus E, Cole MD. 2005. Polycomb mediates Myc autorepression and its transcriptional control of many loci in Drosophila. Genes Dev 19: 2941-2946.

Goodliffe JM, Cole MD, Wieschaus E. 2007. Coordinated regulation of Myc trans-activation targets by Polycomb and the Trithorax group protein Ash1. BMC Mol Biol 8: 40 .

Goyette M, Petropoulos CJ, Shank PR, Fausto N. 1984. Regulated transcription of c-Ki-ras and c-myc during compensatory growth of rat liver. Mol Cell Biol 4: 1493-1498.

Grewal SS, Li L, Orian A, Eisenman RN, Edgar BA. 2005. Myc-dependent regulation of ribosomal RNA synthesis during Drosophila development. Nat Cell Biol 7: 295302.

Harris RE, Pargett M, Sutcliffe C, Umulis D, Ashe HL. 2011. Brat promotes stem cell differentiation via control of a bistable switch that restricts BMP signaling. Dev Cell 20: $72-83$.

Herranz H, Perez L, Martin FA, Milan M. 2008. AWingless and Notch double-repression mechanism regulates $\mathrm{G}_{1}-\mathrm{S}$ transition in the Drosophila wing. EMBO J 27: $1633-$ 1645.

Herranz H, Hong X, Perez L, Ferreira A, Olivieri D, Cohen SM, Milan M. 2010. The miRNA machinery targets Mei-P26 and regulates Myc protein levels in the Drosophila wing. EMBO J 29: 1688-1698.

Hulf T, Bellosta P, Furrer M, Steiger D, Svensson D, Barbour A, Gallant P. 2005. Whole-genome analysis reveals a strong positional bias of conserved dMyc-dependent Eboxes. Mol Cell Biol 25: 3401-3410.

Jin Z, Kirilly D, Weng C, Kawase E, Song X, Smith S, Schwartz J, Xie T. 2008. Differentiation-defective stem cells outcompete normal stem cells for niche occupancy in the Drosophila ovary. Cell Stem Cell 2: 39-49.

* Johnston LA. 2013. Socializing with MYC: Cell competition in development and as a model for premalignant cancer. Cold Spring Harb Perspect Med doi: 10.1101/cshper spect.a014274.

Johnston LA, Prober DA, Edgar BA, Eisenman RN, Gallant P. 1999. Drosophila myc regulates cellular growth during development. Cell 98: 779-790.

Kamoshida Y, Fujiyama-Nakamura S, Kimura S, Suzuki E, Lim J, Shiozaki-Sato Y, Kato S, Takeyama K. 2012. Ecdysone receptor (EcR) suppresses lipid accumulation in the Drosophila fat body via transcription control. Biochem Biophys Res Commun 421: 203-207.

Kappes G, Deshpande G, Mulvey BB, Horabin JI, Schedl P. 2011. The Drosophila Myc gene, diminutive, is a positive regulator of the Sex-lethal establishment promoter, SxlPe. Proc Natl Acad Sci 108: 1543-1548.

Killip LE, Grewal SS. 2012. DREF is required for cell and organismal growth in Drosophila and functions downstream of the nutrition/TOR pathway. Dev Biol 371: 191-202.

Legent K, Steinhauer J, Richard M, Treisman JE. 2012. A screen for X-linked mutations affecting Drosophila photoreceptor differentiation identifies casein kinase $1 \alpha$ as an essential negative regulator of wingless signaling. Genetics 190: 601-616.

Li F, Xiang Y, Potter J, Dinavahi R, Dang CV, Lee LA. 2006. Conditional deletion of c-myc does not impair liver regeneration. Cancer Res 66: 5608-5612.

Li L, Edgar B, Grewal S. 2010. Nutritional control of gene expression in Drosophila larvae via TOR, Myc and a novel cis-regulatory element. BMC Cell Biol 11: 7. 
Lin CY, Loven J, Rahl PB, Paranal RM, Burge CB, Bradner JE, Lee TI, Young RA. 2012. Transcriptional amplification in tumor cells with elevated c-Myc. Cell 151: 56-67.

Loo LW, Secombe J, Little JT, Carlos LS, Yost C, Cheng PF, Flynn EM, Edgar BA, Eisenman RN. 2005. The transcriptional repressor dMnt is a regulator of growth in Drosophila melanogaster. Mol Cell Biol 25: 7078-7091.

Maines JZ, Stevens LM, Tong X, Stein D. 2004. Drosophila $\mathrm{dMyc}$ is required for ovary cell growth and endoreplication. Development 131: 775-786.

Marinho J, Casares F, Pereira PS. 2011. The Drosophila Nol12 homologue viriato is a dMyc target that regulates nucleolar architecture and is required for $\mathrm{dMyc}$-stimulated cell growth. Development 138: 349-357.

Marshall L, Rideout EJ, Grewal SS. 2012. Nutrient/TORdependent regulation of RNA polymerase III controls tissue and organismal growth in Drosophila. EMBO J 31: 1916-1930.

Mitchell NC, Johanson TM, Cranna NJ, Er ALJ, Richardson HE, Hannan RD, Quinn LM. 2010. Hfp inhibits Drosophila myc transcription and cell growth in a TFIIH/Haydependent manner. Development 137: 2875-2884.

Moberg KH, Mukherjee A, Veraksa A, Artavanis-Tsakonas S, Hariharan IK. 2004. The Drosophila F box protein archipelago regulates $\mathrm{dMyc}$ protein levels in vivo. Curr Biol 14: $965-974$.

Montero L, Muller N, Gallant P. 2008. Induction of apoptosis by Drosophila Myc. Genesis 46: 104-111.

Morata G, Ripoll P. 1975. Minutes: Mutants of Drosophila autonomously affecting cell division rate. Dev Biol 42: 211-221.

Moreno E, Basler K. 2004. dMyc transforms cells into supercompetitors. Cell 117: 117-129.

Neto-Silva RM, de Beco S, Johnston LA. 2010. Evidence for a growth-stabilizing regulatory feedback mechanism between Myc and Yorkie, the Drosophila homolog of Yap. Dev Cell 19: 507-520.

Neumuller RA, Betschinger J, Fischer A, Bushati N, Poernbacher I, Mechtler K, Cohen SM, Knoblich JA. 2008. MeiP26 regulates microRNAs and cell growth in the Drosophila ovarian stem cell lineage. Nature 454: 241-245.

Nie Z, Hu G, Wei G, Cui K, Yamane A, Resch W, Wang R, Green DR, Tessarollo L, Casellas R, et al. 2012. c-Myc is a universal amplifier of expressed genes in lymphocytes and embryonic stem cells. Cell 151: 68-79.

Orian A, Van Steensel B, Delrow J, Bussemaker HJ, Li L, Sawado T, Williams E, Loo LW, Cowley SM, Yost C, et al. 2003. Genomic binding by the Drosophila Myc, Max, Mad/Mnt transcription factor network. Genes Dev 17: 1101-1114.

Orian A, Delrow JJ, Rosales Nieves AE, Abed M, Metzger D, Paroush Ze, Eisenman RN, Parkhurst SM. 2007. A Myc Groucho complex integrates EGF and Notch signaling to regulate neural development. Proc Natl Acad Sci 104: 15771-15776.

* O'Shea JM, Ayer DE. 2013. Coordination of nutrient availability and utilization by MAX- and MLX-centered transcription networks. Cold Spring Harb Perspect Med 3: a014258.

Pan D. 2010. The hippo signaling pathway in development and cancer. Dev Cell 19: 491-505.
Parisi F, Riccardo S, Daniel M, Saqcena M, Kundu N, Pession A, Grifoni D, Stocker H, Tabak E, Bellosta P. 2011. Drosophila insulin and target of rapamycin (TOR) pathways regulate GSK-3 $\beta$ activity to control Myc stability and determine Myc expression in vivo. BMC Biol 9: 65.

Pennetier D, Oyallon J, Morin-Poulard I, Dejean S, Vincent A, Crozatier M. 2012. Size control of the Drosophila hematopoietic niche by bone morphogenetic protein signaling reveals parallels with mammals. Proc Natl Acad Sci 109: 3389-3394.

Perrin L, Benassayag C, Morello D, Pradel J, Montagne J. 2003. Modulo is a target of Myc selectively required for growth of proliferative cells in Drosophila. Mech Dev 120: 645-655.

Peyrefitte S, Kahn D, Haenlin M. 2001. New members of the Drosophila Myc transcription factor subfamily revealed by a genome-wide examination for basic helix-loop-helix genes. Mech Dev 104: 99-104.

Pierce SB, Yost C, Britton JS, Loo LW, Flynn EM, Edgar BA, Eisenman RN. 2004. dMyc is required for larval growth and endoreplication in Drosophila. Development 131: 2317-2327.

Pierce SB, Yost C, Anderson SAR, Flynn EM, Delrow J, Eisenman RN. 2008. Drosophila growth and development in the absence of dMyc and dMnt. Dev Biol 315: $303-$ 316.

Prober DA, Edgar BA. 2000. Ras1 promotes cellular growth in the Drosophila wing. Cell 100: 435-446.

Quinn LM, Dickins RA, Coombe M, Hime GR, Bowtell DD, Richardson H. 2004. Drosophila Hfp negatively regulates dmyc and stg to inhibit cell proliferation. Development 131: $1411-1423$.

Rajan A, Perrimon N. 2011. Drosophila as a model for interorgan communication: Lessons from studies on energy homeostasis. Dev Cell 21: 29-31.

Rajan A, Perrimon N. 2012. Drosophilacytokine unpaired 2 regulates physiological homeostasis by remotely controlling insulin secretion. Cell 151: 123-137.

Reddy BVVG, Irvine KD. 2011. Regulation of Drosophila glial cell proliferation by Merlin-Hippo signaling. Development 138: 5201-5212.

Rhiner C, Diaz B, Portela M, Poyatos JF, Fernandez-Ruiz I, Lopez-Gay JM, Gerlitz O, Moreno E. 2009. Persistent competition among stem cells and their daughters in the Drosophila ovary germline niche. Development 136: 995-1006.

Rideout EJ, Marshall L, Grewal SS. 2012. Drosophila RNA polymerase III repressor Maf1 controls body size and developmental timing by modulating tRNAiMet synthesis and systemic insulin signaling. Proc Natl Acad Sci 109: 1139-1144.

Ruggero D. 2012. Revisiting the nucleolus: From marker to dynamic integrator of cancer signaling. Sci Signal 5: pe38.

Sansom OJ, Meniel VS, Muncan V, Phesse TJ, Wilkins JA, Reed KR, Vass JK, Athineos D, Clevers H, Clarke AR 2007. Myc deletion rescues Apc deficiency in the small intestine. Nature 446: 676-679.

Schreiber-Agus N, Stein D, Chen K, Goltz JS, Stevens L, DePinho RA. 1997. Drosophila Myc is oncogenic in mammalian cells and plays a role in the diminutive phenotype. Proc Natl Acad Sci 94: 1235-1240. 
P. Gallant

Schulein C, Eilers M. 2009. An unsteady scaffold for Myc. EMBO J 28: 453-454.

Schwamborn JC, Berezikov E, Knoblich JA. 2009. The TRIM-NHL protein TRIM32 activates microRNAs and prevents self-renewal in mouse neural progenitors. Cell 136: $913-925$.

Schwinkendorf D, Gallant P. 2009. The conserved Myc box 2 and Myc box 3 regions are important, but not essential, for Myc function in vivo. Gene 436: 90-100.

Secombe J, Li L, Carlos L, Eisenman RN. 2007. The Trithorax group protein Lid is a trimethyl histone $\mathrm{H} 3 \mathrm{~K} 4$ demethylase required for $\mathrm{dMyc}$-induced cell growth. Genes Dev 21: 537-551.

Smith-Bolton RK, Worley MI, Kanda H, Hariharan IK. 2009. Regenerative growth in Drosophila imaginal discs is regulated by Wingless and Myc. Dev Cell 16: 797-809.

Song Y, Lu B. 2011. Regulation of cell growth by Notch signaling and its differential requirement in normal vs. tumor-forming stem cells in Drosophila. Genes Dev 25: 2644-2658.

Steiger D, Furrer M, Schwinkendorf D, Gallant P. 2008. Maxindependent functions of Myc in Drosophila. Nature Gen 40: $1084-1091$.

Teleman AA, Hietakangas V, Sayadian AC, Cohen SM. 2008. Nutritional control of protein biosynthetic capacity by insulin via Myc in Drosophila. Cell Metab 7: 21-32.
Tennessen JM, Thummel CS. 2011. Coordinating growth and maturation: Insights from Drosophila. Curr Biol 21: R750-R757.

Thao DT, Seto H, Yamaguchi M. 2008. Drosophila Myc is required for normal DREF gene expression. Exp Cell Res 314: $184-192$.

Toyoshima M, Howie HL, Imakura M, Walsh RM, Annis JE, Chang AN, Frazier J, Chau BN, Loboda A, Linsley PS, et al. 2012. Functional genomics identifies therapeutic targets for MYC-driven cancer. Proc Natl Acad Sci 109: 9545-9550.

Trumpp A, Refaeli Y, Oskarsson T, Gasser S, Murphy M, Martin GR, Bishop JM. 2001. c-Myc regulates mammalian body size by controlling cell number but not cell size. Nature 414: 768-773.

* Wiese K, Walz S, von Eyss B, Wolf E, Athineos D, Sansom O, Eilers M. 2013. The role of MIZ1 in MYC-dependent tumorigenesis. Cold Spring Harb Perspect Med doi: 10.1101/cshperspect.a014290.

Wu DC, Johnston LA. 2010. Control of wing size and proportions by Drosophila Myc. Genetics 184: 199-211.

Zaffran S, Chartier A, Gallant P, Astier M, Arquier N, Doherty D, Gratecos D, Semeriva M. 1998. A Drosophila RNA helicase gene, pitchoune, is required for cell growth and proliferation and is a potential target of d-Myc. Development 125: 3571-3584. 


\title{
$\&_{\mathrm{CSH}}^{\infty} \&$ Cold Spring Harbor

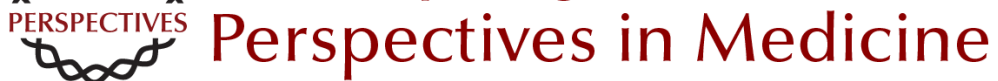

\section{Myc Function in Drosophila}

\author{
Peter Gallant
}

Cold Spring Harb Perspect Med 2013; doi: 10.1101/cshperspect.a014324

Subject Collection MYC and the Pathway to Cancer

\section{MYC Cofactors: Molecular Switches Controlling Diverse Biological Outcomes Stephen R. Hann}

MYC Association with Cancer Risk and a New Model of MYC-Mediated Repression Michael D. Cole

MYC and the Art of MicroRNA Maintenance James N. Psathas and Andrei Thomas-Tikhonenko

MYC Activation Is a Hallmark of Cancer Initiation and Maintenance Meital Gabay, Yulin Li and Dean W. Felsher

MYC and Mitochondrial Biogenesis Fionnuala Morrish and David Hockenbery

Synthetic Lethal Screens as a Means to Understand and Treat MYC-Driven Cancers Silvia Cermelli, In Sock Jang, Brady Bernard, et al.

An Overview of MYC and Its Interactome Maralice Conacci-Sorrell, Lisa McFerrin and Robert N. Eisenman

Socializing with MYC: Cell Competition in Development and as a Model for Premalignant Cancer

Laura A. Johnston
MYC and the Control of Apoptosis Steven B. McMahon

Therapeutic Strategies to Inhibit MYC Michael R. McKeown and James E. Bradner

MYC and the Control of DNA Replication David Dominguez-Sola and Jean Gautier MYC Regulation of Cell Growth through Control of Transcription by RNA Polymerases I and III Kirsteen J. Campbell and Robert J. White

MYC Degradation Amy S. Farrell and Rosalie C. Sears

MYC and Transcription Elongation Peter B. Rahl and Richard A. Young

c-MYC-Induced Genomic Instability Alexandra Kuzyk and Sabine Mai

Oncogenic Mechanisms in Burkitt Lymphoma Roland Schmitz, Michele Ceribelli, Stefania Pittaluga, et al.

For additional articles in this collection, see http://perspectivesinmedicine.cshlp.org/cgi/collection/ 